\title{
Hábitat campesino desde un enfoque integral: análisis de una experiencia de diseño colectivo
}

\author{
Peasant habitat from an integral approach: analysis of a collective \\ design experience
}

\section{Habitat camponês sob uma abordagem integral: análise de uma experiência coletiva de design}

\section{Noelia Verónica Cejas}

Licenciatura en Comunicación Social, Doctora en Estudios Sociales de América Latina. Investigadora Asociada Centro Experimental de Vivienda Económica, Argentina.

Email: noelia_cejas@outlook.com

\section{María Rosa Mandrini}

Arquitecta, Doctora en Arquitectura y Urbanismo.

Investigadora Asociada Centro de Investigaciones y Estudios sobre Cultura y Sociedad, Universidad Nacional de Córdoba, Argentina.

Email: mrmandrini8@gmail.com

\section{Julián González Laria}

Arquitecto, Doctorante.

Investigador Asociado Centro Experimental de Vivienda Económica, Argentina.

Email: juliangonzalezlaria@gmail.com

\section{RESUMEN}

El artículo presenta las principales definiciones de política públicas para el hábitat campesino en Córdoba (Argentina), como contexto general de indagación, para pasar a analizar una experiencia concreta de una comunidad en la que la implementación de esta política tuvo la particularidad de estar entrelazada con un proceso de investigación-acción a cargo del equipo autor del artículo y representantes de otras instituciones científico-técnicas. Por medio de esta experiencia nos preguntamos por las posibilidades de componer una perspectiva integral de abordaje al hábitat campesino. El artículo presenta las singularidades de una experiencia colectiva de transformación del hábitat rural de un grupo campesino, Agua Viva, (La Patria, Cba) en la cual se revisan las definiciones tecnológicas con que el Estado se aproximó a estos territorios y al mismo tiempo, se despliega una experiencia de diálogo de saberes, integrando saberes locales con una mirada interdisciplinar para el diseño de un espacio socio-productivo comunitario.

Palabras clave: hábitat, tecnología social, diálogo de saberes. 


\section{ABSTRACT}

The article presents the main public policy definitions for the peasant habitat in Córdoba (Argentina), as a general context of inquiry, to analyze a concrete experience of a community in which the implementation of this policy had the particularity of being intertwined with an action research process by the author of the article and representatives of other scientific and technical institutions. Through this experience we ask ourselves about the possibilities of composing an integral perspective of approach to the peasant habitat. The article presents the singularities of a collective experience of transformation of the rural habitat of a peasant group, Agua Viva, (La Patria, $\mathrm{Cba}$ ) in which the technological definitions with which the State approached these territories and at the same time are reviewed, an experience of knowledge dialogue unfolds, integrating local knowledge with an interdisciplinary look for the design of a community socio-productive space.

Keywords: habitat, social technology, knowledge dialogue.

\section{RESUMO}

O artigo apresenta as principais definições de políticas públicas para o habitat camponês em Córdoba (Argentina), como um contexto geral de investigação, para analisar uma experiência concreta de uma comunidade na qual a implementação dessa política teve a particularidade de estar entrelaçada com um processo de pesquisa-ação pelo autor do artigo e representantes de outras instituições científicas e técnicas. Com essa experiência, nos perguntamos sobre as possibilidades de compor uma perspectiva integral de abordagem do habitat camponês. O artigo apresenta as singularidades de uma experiência coletiva de transformação do habitat rural de um grupo camponês Agua Viva, (La Patria, Cba), no qual são revisadas as definições tecnológicas com as quais o Estado abordou esses territórios e, ao mesmo tempo, são revisadas, desdobra-se uma experiência de diálogo do conhecimento, integrando o conhecimento local com um olhar interdisciplinar para o desenho de um espaço sócio-produtivo da comunidade.

Palabras chave: habitat, tecnologia social, diálogo sobre conhecimento. 


\section{Introducción}

En la provincia de Córdoba, desde comienzos del año 2000 se desarrolla el denominado "Plan de Desarrollo del Noroeste". Se trata de una serie de acciones, impulsadas desde distintas reparticiones de gobierno, destinadas a comunidades rurales y tendientes a atender diversos planos: por un lado, la construcción de obras de equipamiento público -como dispensarios y escuelas- y la construcción de viviendas nuevas. Asimismo, el plan abarca infraestructura de caminos, servicios de agua y electricidad por vía de sistemas fotovoltaicos, entre otros.

En publicaciones oficiales y periodísticas que las referencian', se observa una lectura diagnóstica cualitativa amplia del hábitat rural, que incluye la necesidad de apuntalar las actividades productivas de los lugareños, abocados a la producción primaria mayormente. En lo que respecta a lo habitacional, se hace una lectura de variables más acotadas, especialmente asentada en la dimensión arquitectónica de la vivienda rural y más puntualmente observando problemáticas asociadas a las técnicas constructivas locales, planteándolas en asociación a patologías como la Enfermedad de Chagas. Entre las técnicas constructivas asociadas a patologías podemos señalar el adobe y quincha, cuya característica común es la utilización de tierra y materiales obtenidos del entorno natural próximo para su elaboración. De manera más específica podemos decir que se conoce con el término adobe al bloque de tierra moldeado, secado al sol, que se utiliza como mampuesto para la construcción de muros o la realización de cúpulas o bóvedas (Tomassi y Bellmann, 2018); asimismo el término Quincha refiere a un sistema constructivo basado en el uso de estructuras de madera, caña u otras fibras vegetales, azotadas con barro para la materialización de cerramientos. Esta técnica forma parte de las denominadas técnicas de "entramados" o "técnicas mixtas" que se caracterizan principalmente por la presencia de más de un material, donde la resistencia estructural depende fundamentalmente en marcos de madera y donde el entramado vegetal interno funciona como estructura auxiliar destinada a sostener y consolidar el relleno de barro en el muro (Garzón, 2011 en Tomassi y Bellmann, 2018). El reconocimiento de estas técnicas constructivas, atendiendo a su carácter situado (tanto en el plano materia - de los recursos disponibles- como simbólico -por tratarse de un saber ancestral vernáculo) nos permite mirar más allá de las discusiones que pretenden centrar en el tipo de material las discusiones en torno al factor de riesgo antes que en la calidad constructiva de la vivienda. Junto a otros/as autores/as consideramos que esa mirada "puede acarrear un sesgo muy importante sobre ciertas decisiones o argumentaciones como, por ejemplo, la elección de técnicas de construcción entre opciones disponibles o conducir indebidamente a la estigmatización del conocimiento cultural de una comunidad" (Rolón, Olivarez, Dorado y Varela Freire, 2016, p. 66)

El planteo general del Plan de Desarrollo del Noroeste procura estimular el desarrollo de una de las regiones con mayor índice de pobreza en la provincia ${ }^{2}$, interviniendo en aspectos que remiten al acondicionamiento del patrimonio material de las comunidades: acceso a energías

1. Entre ellos, podemos señalar: documentos oficiales del Estado provincial (planes, programas y proyectos diseñados e implementados por las distintas gestiones provinciales), publicaciones institucionales del estado provincial (publicados en medios oficiales, esencialmente su página web y cuentas oficiales en redes) e información publicada en medios de comunicación de circulación local (privilegiamos los medios de prensa gráfica local, La Voz del Interior, por ejemplo, y portales web de medios locales, como Cadena 3, otro ejemplo).

Como hemos señalado anteriormente, bajo la denominación de "Plan de Desarrollo del Noroeste", se articulan acciones tendientes a atender diversos planos: por un lado, la construcción de obras de equipamiento público como dispensarios y escuelas, y por otro, la construcción de viviendas nuevas. También abarca infraestructura de caminos, servicios de agua y electricidad. Por todo esto, se presenta al Plan como una experiencia transversal que articula diversas dependencias. Esto supone además la multiplicidad de productores de contenidos en torno al Plan.

2. Los datos publicados por INDEC en el año 2010, indican que en la provincia de Córdoba el mayor índice de población con $\mathrm{NBI}$ se encuentra en áreas rurales. Específicamente, la región del noroeste cordobés es la zona más relegada en cuanto a indicadores de productividad, según muestra la Dirección General de Estadísticas y Censos de la provincia de Córdoba en el último informe de producto bruto regional (2015). 
alternativas, agua potable y erradicación de las viviendas afectadas o que presenten probabilidades de ser afectadas por el vector de la Enfermedad de Chagas, denominadas como viviendas ranchos 3 .

En ese marco, signado por transformaciones espaciales, sociales y políticas que atraviesan comunidades campesinas, nos preguntamos - desde una perspectiva orientada a abordar de manera integral el hábitat- por los alcances de estas intervenciones territoriales, concretamente las de erradicación de viviendas rancho y las de acceso a la energía. Focalizamos allí, no sólo porque entendemos que estas viviendas rurales constituyen parte del patrimonio material modesto (ICOMOS, 1965), sino que, además, representan parte del patrimonio inmaterial, al traer aparejadas una serie de saberes y técnicas constructivas, transmitidas de generación en generación, que se han ido modificando y perfeccionando a través de los años a partir de la redefinición de las necesidades en la construcción del hábitat propio. Estas tecnologías endógenas, mantienen una condición esencial de independencia, por estar basadas en conocimientos y materiales disponibles libremente en el lugar, que las exógenas desplazan. Al mismo tiempo, y con operaciones interpretativas similares, observamos que algunas políticas de acceso a la energía presentan definiciones un tanto desajustadas de aquello que las comunidades requieren para encauzar el fortalecimiento de sus economías.

El diseño metodológico desde el cual abordamos la cuestión procura acercarnos a comunidades campesinas e instituciones estatales presentes en el territorio para plantear desde allí una revisión crítica de los alcances de las definiciones con que se impacta en los territorios. Esto no transcurre en diálogos abstractos, ni tampoco se despliega por medio de técnicas de entrevista o encuesta -caracterizadas por la posibilidad de enunciar ordenadamente la experiencia y la posición crítica-sino que se produce habilitando espacios concretos de diseño colectivo, procurando revisar desde allí las posibilidades materiales, los saberes disponibles, los soportes discursivos, las definiciones subyacentes y los horizontes de deseo.

En este artículo revisamos una experiencia de ese orden, desarrollada en la provincia de Córdoba, Argentina, junto a un grupo de productores campesinos, denominado Agua Viva, en el paraje "La Patria". Allí participamos también representantes del sector científico tecnológico nacional, como lo somos quienes formamos parte de CONICET (Consejo Nacional de Investigaciones Científicas y Técnicas) e INTA (Instituto Nacional de Tecnología Agropecuaria) y también representantes del gobierno provincial por el MAASP (Ministerio de Agua, Ambiente y Servicios Públicos de la provincia de Córdoba; concretamente, el responsable de la implementación del programa de acceso a la energía solar) y del gobierno comunal (responsable de la ejecución del plan habitacional).

A fin de realizar una presentación y análisis de la experiencia de manera ordenada, presentamos 3 ejes conceptuales que nos permitirán profundizar en la riqueza de la experiencia y cristalizar algunos aprendizajes y nuevos interrogantes.

\section{Categorías de análisis}

Como perspectiva central, recuperamos los aportes del sociólogo portugués Boaventura de Sousa Santos (2009) para pensar y accionar en torno a otros modos de producción de conocimiento. En ese sentido, una de las categorías acuñadas por Santos, diálogo de saberes, nos permite, como primer eje de análisis comprender y desplegar prácticas situadas de desarrollo tecnológico capaces de integrar campos de experiencia diversos, sin que sea el saber académico el que ordene o jerarquice los otros saberes en presencia.

3. Consideramos a estas viviendas como parte del patrimonio material modesto del lugar, ya que a partir de la Carta de Venecia de 1964 se entiende que "la noción de monumento histórico comprende la creación arquitectónica aislada, así como el conjunto urbano o rural que da testimonio de una civilización particular, de una evolución significativa, o de un acontecimiento histórico. Se refiere no sólo a las grandes creaciones sino también a las obras modestas que han adquirido con el tiempo una significación cultural" (ICOMOS, 1965, p. 1). 
Es decir, partiendo desde el principio de "incompletitud de todo saber" (de Sousa Santos 2006, p.79) es posible reconfigurar las bases desde las cuales abordamos la definición de problemas y soluciones, habilitando una lectura transdisciplinaria, que incorpore los saberes emergentes de los territorios y sus agentes.

Concretamente, en el campo de la producción del hábitat campesino y más específicamente en las acciones emprendidas desde el Estado, no se advierte ni se dialoga con el saber endógeno de las comunidades en estos aspectos. Por el contrario, ese saber es activamente desautorizado, silenciado. Entendemos, de manera contraria, que existe una trama de sentidos y conceptualizaciones íntimamente vinculadas con el habitar estos territorios que hacen de esas estrategias habitacionales un campo de gran valor gnoseológico. Bajo esa perspectiva, el diálogo de saberes busca indagar en esa configuración de conocimientos expresada en una forma espacial y vital que habilite otras resoluciones territoriales.

Para abordar procesos de este tipo, el autor portugués define la instancia de diálogo como un ejercicio de dislocación pragmática de las jerarquías gnoseológicas. Allí, el desafío consiste tanto en penetrar más allá de los límites disciplinares, como en reconstruir un posicionamiento paradigmático situado, habilitado por la expresividad de esos otros saberes (profundizaremos en este aspecto en el apartado metodológico). En ese sentido, como segundo eje de trabajo, entendemos que la búsqueda de resoluciones a partir de esquemas de autonomía son la clave para el pragmatismo en cuestión. Estas formas de desarrollo tecnológico y la comprensión de todo lo que moviliza tal desarrollo, prioriza la jerarquización de aquellos saberes que hacen de las comunidades agentes autónomos (tanto en aspectos constructivos como también productivos y organizacionales) ya que permite subvertir órdenes instituidos de jerarquización entre saberes académicos y saberes emergentes de los territorios. Con esto no indicamos tampoco una romantización de los saberes locales, ni un silenciamiento de los saberes académicos, por el contrario, entendemos que la articulación de ambos puede potenciar el ejercicio autónomo de las prácticas locales y esa es la mayor responsabilidad y compromiso que podemos asumir como académicos/as.

Finalmente, como tercer eje de análisis se plantea el reconocimiento de las materialidades disponibles en la zona, en articulación con el reconocimiento de su inscripción ambiental, a fin de hacer sinergia con los dos aspectos anteriores: la autonomía y el diálogo de saberes situados. En ese sentido, las formas de habitar campesinas plantean modos armónicos con la naturaleza que pueden abrir la discusión sobre la sustentabilidad, leída en clave de ecología política.

\section{Metodología}

Como se mencionó anteriormente, nos proponemos reconocer los alcances de las políticas públicas de hábitat señaladas, a fin de analizar la experiencia concreta de una comunidad en la que la implementación de esta política tuvo la particularidad de estar entrelazada con un proceso de investigación-acción. Por medio de esta experiencia nos preguntamos por las posibilidades de componer una perspectiva integral de abordaje al hábitat campesino. La experiencia colectiva de transformación del hábitat rural que se analiza, toma lugar en La Patria (Córdoba), en la que nos articulamos un grupo campesino, Agua Viva, miembros de un equipo de CONICET (Consejo Nacional de Investigaciones Científicas y Técnicas) e INTA (Instituto Nacional de Tecnología Agropecuaria) y también representantes del gobierno provincial por el MAASP (Ministerio de Agua, Ambiente y Servicios Públicos de la provincia de Córdoba; concretamente, el responsable de la implementación del programa de acceso a la energía solar) y del gobierno comunal (responsable de la ejecución del plan habitacional). A propósito de la implementación de financiamiento estatal para acceso a la energía solar y mejoras edilicias, se planteó un ejercicio de investigación-acción capaz de revisar, desde las definiciones endógenas, aquello que mejor podría definir el carácter integral de la intervención. Así, se centró la atención en 
fortalecer los modos de habitar campesinos, por medio de entender sus prácticas de manera integral (en su concepción residencial, económica-productiva y organizativa). Es decir, a partir de comprender las prácticas productivas como actividades cotidianas y propias de cada familia, se planteó un espacio productivo que respondiera a la costumbre colectiva, centrada en el almacenamiento de su producción y no en su elaboración, actividad que realizan de modo independiente, en cada vivienda.

El diseño de investigación, centrado en la perspectiva cualitativa-constructivista de investigación-acción, dio lugar a una práctica recursiva y dialógica entre los actores partícipes de la experiencia, quedando esto plasmado en las notas de campo, fotografías de la experiencia de diseño colectivo y sucesivos rediseños de plantas arquitectónicas y detalles constructivos que suscitó el devenir de la experiencia.

La metodología adoptada con el grupo Agua Viva se planteó a partir de las siguientes premisas: 1) diálogo de saberes, 2) resoluciones desde la autonomía, 3) reconocimiento de materialidades disponibles y su inscripción ambiental.

La perspectiva de investigación se basa en el desarrollo situado de tecnología social, desde un enfoque integral a partir del diálogo de saberes (de Sousa Santos, 2009). El despliegue de formas alternativas de producción de conocimiento, promoviendo instancias de diálogo entre saberes, de recupero de experiencias -individuales y colectivas, evocadas por vía oral o corporal, como expresión del acervo de memoriapermite enriquecer tanto la comprensión de las problemáticas como también la base gnoseológica con que se construyen soluciones.

Metodológicamente, cabe señalar que el diálogo de saberes, tomado en su definición más literal, produce una escena imposible de sostener; no importa que tan pretendidamente persuasiva pueda ser la invitación a una producción participativa del conocimiento, en tanto reproduzcamos prácticas de extractivismo académico, guiadas por objetivos ajenos al de las comunidades con las que se investiga, no será posible transformar ese orden dominante.
Walter Mignolo (2003) convoca a cambiar los términos de la conversación y no sólo los contenidos. En ello, entendemos que subyace un cuestionamiento a las metodologías con que se instaura la relación sujeto conocido-sujeto cognoscente (Vasilachis de Gialdino, 2007) en los territorios, aspecto ineludible e inescindible de este planteo teórico y epistémico.

Esto supone desmantelar un dispositivo que se estableció desde la academia, que aun cuando busca atender problemáticas sentidas de injusticia social, no necesariamente logra detener la recurrente producción de subalternidades, a través de lógicas que no hacen más que reproducir la jerarquía con que se diferencian los campos de experiencia y saber: el extractivismo epistémico (Grosfoguel, 2015) o académico, que encierra la idea de informante, entrevistado o entrevistada, encuestado o encuestada, en fin, la innegable asimetría de ser "sujeto por conocer" (Vasilachis de Gialdino, 2007, p.50) o la utilitaria relación de dominación en la que sólo se espera del otro u otra obediencia ante el saber "legítimo".

De esta manera, esta perspectiva implica un diseño recursivo entre procesos de producción de conocimiento emergentes del territorio, con las comunidades con las que se trabaja, y desarrollo de conocimiento llamado "de gabinete", por denominar los espacios tradicionales de producción de conocimiento científico. Este trabajo en espacios de encuentro permite el desarrollo de tecnologías situadas y progresivos aprendizajes de toda la comunidad involucrada. Así, el conocimiento deviene contextual, dinámico $\mathrm{y}$, especialmente, relacional.

El proceso se organizó en etapas: 1) mediante diversos encuentros multiactorales e interdisciplinarios en el territorio (conformados por el grupo Agua Viva de La Patria, personal técnico del INTA, actores gubernamentales, constructores locales y el equipo de CONICET) se dialogó sobre las formas de vida, cultura, costumbres de la comunidad desde diferentes aproximaciones, arrojando como resultado inicial un mapeo o diagnóstico de las necesidades del grupo. 2) Luego se realizó con la misma metodología -dialógica- el diseño funcional y 

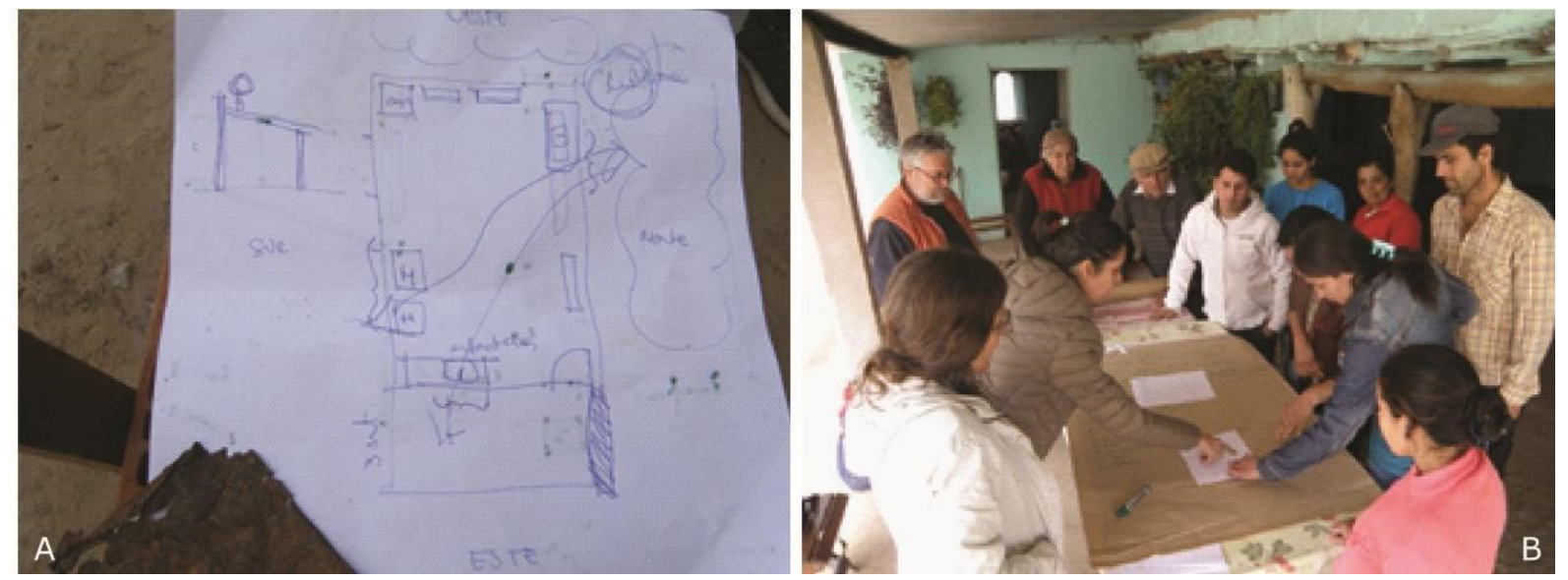

Figura 1 Encuentros multiactorales para definición de criterios y deseos sobre el futuro espacio de trabajo.

espacial del espacio a construir. 3) Seguidamente se realizó la resolución técnica constructiva de cada componente arquitectónico para ese espacio y 4) finalmente la construcción del mismo por parte del grupo con apoyo técnico interno del INTA y apoyo técnico externo del equipo de CONICET. En la presentación de resultados se amplían detalles sobre la implementación de estas metodologías.

\section{Resultados}

Se presentan los resultados empíricos, en diálogo con las reflexiones teóricas, dentro del proceso de transformación socio-espacial que significó la construcción de un salón de uso comunitario con el grupo campesino Agua Viva.

\section{Resultados de la etapa 1: Mapeo colectivo y diagnóstico de necesidades}

Posicionándonos desde el eje de análisis de diálogo de saberes, reconocemos que el proceso se abordó desde el relato colectivo, sobre la función espacial, a fin de definir las actividades que debería contener, la cantidad de familias que lo habitarían, la frecuencia de uso y el tipo de maquinarias e insumos que debería albergar.

En esta etapa pudo observarse que, como criterios funcionales, se prefiguraba un espacio de trabajo colectivo en el cual almacenar los productos derivados del monte que la comunidad produce, en un lugar fresco y con el agregado de heladeras para su conservación. A su vez debería contener un espacio para la exposición de los productos y tener un ingreso directo para las familias que residen a mayor distancia, con el fin de almacenar su producción en un espacio común. Al mismo tiempo se incorporó al diseño un sistema de recolección de agua de Iluvia para almacenaje en una cisterna.

En términos de funcionalidad, se acordó que la sala debería contemplar espacio para: almacenaje del fondo rotatorio de insumos comunes, sellado al vacío de quesos y maduración dentro de las heladeras, almacenaje, exposición y venta de productos de todas las familias -en frascos de vidrio y potes plásticos etiquetados- acorde a protocolos acordados colectivamente. También se definió que la elaboración de quesos, dulces y arropes sería realizada por cada familia y de manera diaria; por tal motivo la sala no debería contemplar espacio para la elaboración, sino para la conservación. En ese sentido, se comprende que los procesos productivos de este tipo se inscriben en la escala doméstica, porque requieren muchas horas durante las cuales, al mismo tiempo, las trabajadoras realizan otros quehaceres domésticos simultáneos.

El relato colectivo, el lenguaje gráfico y el corporal, dio lugar y expresión a una copresencia de conocimientos contemporáneos: los/as campesinos/as aportaron conocimientos sobre el modo de uso de ese espacio, la persona con formación en técnica agrónoma sobre el almacenamiento adecuado de la producción y los/as técnicos/as de CONICET en cuanto a 
las dimensiones del espacio y la asignación colectiva de sentido, entablando un interconocimiento, aprendiendo de otros saberes, sin retirar el propio.

\section{Resultados de la etapa 2: Diseño arquitectónico colectivo y replanteo}

El diseño funcional de la sala de almacenamiento se realizó en base a las materialidades disponibles en la zona, en articulación con el reconocimiento de su inscripción ambiental, persiguiendo una de las premisas de diseño: mantener una baja temperatura interior, apropiada para la conservación de los productos alimenticios.

Se trabajó sobre la idea de pluralidad de conocimientos, incorporando los saberes que aporten de forma pragmática a cada problema presentado. Este concepto, presentado anteriormente en el apartado 1.1, remite al ejercicio de dislocación pragmática de jerarquías gnoseológicas. Esto permitió hacer foco en los modos de construir campesinos, en las formas de usar los materiales históricamente aprendidas, en comparación a los materiales y técnicas usadas en la actualidad y los costos-beneficios de cada decisión tecnológica en diversos niveles (cultural, económico, ambiental).

En ese sentido, las instancias de diálogo con las comunidades nos permitieron advertir criterios disímiles en la valoración individual de las tecnologías disponibles, habilitando una lectura no homogénea del plano material. En la experiencia, algunas personas destacaron la simpleza que remite a las tecnologías endógenas y otras ponderaron el bajo nivel de mantenimiento que requieren las soluciones tecnológicas a base de mampuestos, prefiriendo así diferentes resoluciones tecnológicas. Se observa que en el primer grupo, están los/ as habitantes con más años de residencia en el lugar y que han permanecido casi toda su vida en la zona, mientras que quienes se desempeñan también en otras actividades en el contexto urbano actualmente, se encuentran en el segundo grupo.

Para consensuar el diseño espacial-funcional se realizaron dibujos en escala 1:100 del equipamiento y maquinarias necesarias y a partir de ubicarlos en el espacio se logró definir la superficie adecuada para la sala, detectando que podría tener una superficie cubierta menor a la imaginada inicialmente. El diseño resultante fue un espacio compacto, suficiente para el trabajo de envasado y almacenamiento.

En la Figura 2, se observa la necesidad de libre acceso por parte de las familias participantes que viven alejadas del sector elegido para la construcción, plasmada en una galería de ingreso.

En cuanto al factor climático del lugar, se conversó sobre las percepciones de los lugareños en las distintas épocas del año. Trabajando sobre conceptos del diseño bioclimático, así como sobre el reconocimiento de su inscripción ambiental sobre las formas de aprovechamiento de la energía natural para lograr la situación térmica deseada; mantener una baja temperatura estable durante todo el año, apropiada al uso funcional del espacio y la conservación de alimentos. Para lograrlo se trabajó cada orientación de fachada de un modo diferente: la cara norte se ubicó en las cercanías de una cortina de árboles medianos, capaces de proteger de la radiación solar en las horas más extremas; al este, se realizó una galería de protección, ya que los vientos cálidos noreste son los más frecuentes y fuertes; en la cara sur, se tuvo en cuenta la presencia de dos aperturas de ventanas altas para lograr ventilación cruzada y eliminar el calor interior rápidamente; con respecto al oeste, que era la fachada en la que no se había logrado protección, se decidió realizar un espacio filtro. Este espacio alberga las baterías correspondientes a los paneles solares que alimentarán las heladeras, resolviendo el tema de la incidencia solar directa en las horas de la tarde hacia el interior de la sala.

Mediante el lenguaje corporal se realizó un replanteo vivo. Es decir, se trasladó el dibujo del diseño consensuado de la sala al terreno donde se comenzaría la construcción. Esta actividad se realizó junto a los habitantes, quienes construirían y habitarían la sala. concretamente, se materializaron los límites de la sala con hilos, a fin de valorar sensorialmente las dimensiones. 
Luego diferentes miembros de la comunidad se ubicaron en ese espacio, representando el lugar que ocuparían los diferentes elementos de la sala: heladera, mesa, ventana, etc. Esto permitió tomar una mayor consciencia del espacio interior que estaría ocupado y el espacio que quedaría destinado a la circulación. Asimismo, esta actividad permitió revisar las orientaciones, proporciones, ubicación de la galería, aberturas y del equipamiento y maquinarias. Este ejercicio nos permitió reflexionar y ajustar algunos detalles colectivamente integrando saberes mediante el movimiento del cuerpo, un lenguaje que subvierte la dominancia del ejercicio discursivo centrado en la conceptualización verbal.

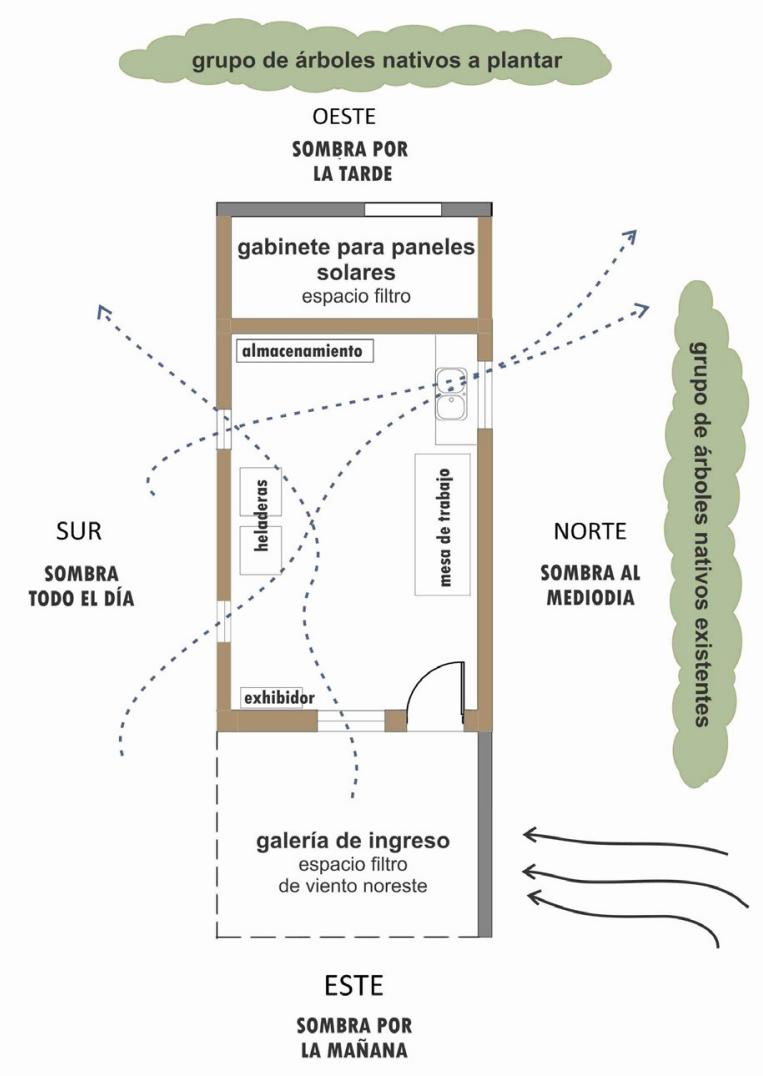

Figura 2 Diseño colectivo resultante de la etapa 2.

\section{Resultados de la etapa 3: Resoluciones tecnológicas-constructivas y proceso de autoconstrucción}

En esta etapa del proceso el diálogo se centró sobre las técnicas constructivas locales y materiales disponibles para la construcción de la sala, así como también se integraron otros conocimientos provenientes de los constructores, de los habitantes y de personal técnico, priorizando las ideas o resoluciones a partir de esquemas de autonomía local, es decir las resoluciones de quienes iban a encargarse de la construcción. Para finalizar en piezas gráficas sencillas y de fácil comprensión técnica, se decidió realizar un sólo detalle constructivo tipo corte "de piso a techo" evidenciando todas las problemáticas posibles y sus pormenores, con el aporte de toda la comunidad. En la figura 3 se observa el gráfico, expresivo y figurativo al mismo tiempo que técnico, emergente del diálogo desde conocimientos plurales. Este mismo elemento cristaliza una herramienta para futuras toma de decisiones autónomas.

Aunque desde el equipo técnico habíamos ensayado algunas resoluciones tecnológicas en base a lo conversado en las etapas anteriores, al llegar al territorio y volver a dialogar colectivamente, se acordó modificar esas propuestas, optando por tecnologías que consideraban más familiares, más apropiadas a los modos de construir locales. Se definió utilizar una estructura independiente de hormigón armado, para los cerramientos se tomaron dos opciones, las paredes norte y sur, de ladrillo cerámico y las este y oeste de adobes (recuperados tras la demolición de una vivienda de 100 años). En todas las decisiones se contemplaron las materialidades disponibles. El muro oeste perteneciente al exterior de las baterías se materializó con ladrillo cerámico hueco. El porqué de estas resoluciones se debió a que la comunidad expresó cierta desconfianza a que las paredes de adobe queden a la intemperie, ya que los adobes utilizados en esta nueva construcción fueron extraídos de una construcción muy antigua, y no estaban seguros de saber cómo se comportarían en esta nueva construcción. Se ponderó así la importancia de preservar estas paredes, con zócalos y aleros de protección, lo cual permitiría prevenir la presencia de humedad y deterioro en las caras externas a causa de la lluvia. También se destacó la construcción de una capa aisladora al muro, que pudiera proteger la construcción del ascenso de humedad proveniente del terreno. Estas definiciones son expresión patente del diálogo de saberes entre la experiencia territorial, constructiva 


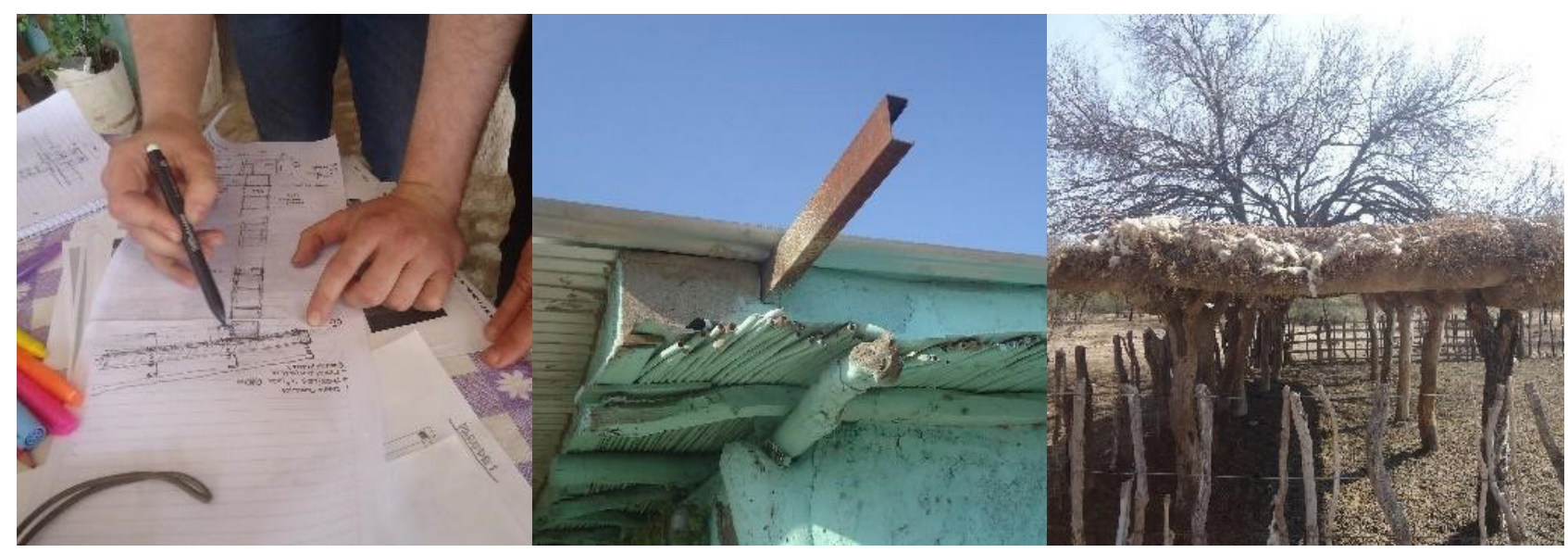

Figura 3 Lenguaje gráfico técnico habilitando el encuentro de conocimientos plurales. Resoluciones locales de techo. Al centro: cañizo, barro y chapa; a la derecha: cañizo, jarilla, barro y lana de oveja

y empírica de la comunidad en diálogo con los aportes disciplinares-arquitectónicos que permitieron resolver terminaciones problemáticas, sentidas por la comunidad. Se incorporan, así, resoluciones de protección/ mejoras constructivas a las formas de construir vernáculas, que se expresan materialmente en diferentes formas de protección (el este, por galería y el oeste, por el espacio filtro, como se puede observar en la figura 2).

Para la resolución del techo y con la finalidad de conservar la baja temperatura interior estable, se definió ensayar un techo aislante mixturando diversas técnicas del lugar en base a un ejemplo conocido: se puede observar en la Fig. 3, en la imagen central, el techo de la casa de un vecino referente del grupo, que para todos/as resulta muy fresca en los momentos de calor extremo. Ese techo está resuelto con caña tacuara y mezcla de barro y en los últimos años le agregaron chapa en la fase exterior para mejorar su aislación hidrófuga. A modo de complemento, se conversó con el grupo sobre la importancia, en términos técnicos, de realizar una cubierta doble (mezcla de barro con aislación en complemento con chapa), señalando que esta genera una sombra adecuada, reduciendo la radiación solar incidente al interior del espacio desde el plano de techo, el de mayor ganancia solar.

La tecnología que se acordó usar como estrategia constructiva en el techo del nuevo salón, integró las experiencias y saberes aprehendidos y algunas variaciones. Se resolvió usar chapa como terminación superficial, como protección de un espacio aislante compuesto de lana de oveja, jarilla y mezcla de barro, observando que se trataba de una resolución usada localmente para brindar sombra a los animales dentro de los corrales (ver Fig. 3, imagen derecha). El criterio que primó fue el peso total del techo; se observó que con esa resolución de techo podría ser más liviano, respecto al que contenía sólo mezcla de barro, por la incorporación de los materiales livianos y aislantes (lana y jarilla) y asegurando la aislación hidrófuga mediante la chapa.

\section{Discusiones}

A partir de la idea del diálogo de saberes, en las tres primeras etapas se logró el abordaje de problemas y soluciones desde la coexistencia de saberes endógenos como exógenos. Es decir, se construyeron acuerdos sobre la necesidad funcional y espacial de las familias, considerando los modos productivos locales tanto como los saberes técnicos en la resolución de ese espacio. Se trabajó desde el reconocimiento de sus modos de construir y de las materialidades disponibles, abarcando las diversas visiones, incluso las diferencias dentro del mismo grupo Agua Viva y tomando en cuenta tanto resoluciones tecnológicas desde el equipo técnico como las aportadas desde la comunidad. Esto desencadenó en la resolución de muros con tecnologías industrializadas en diálogo con tecnologías ancestrales, habilitando otras 

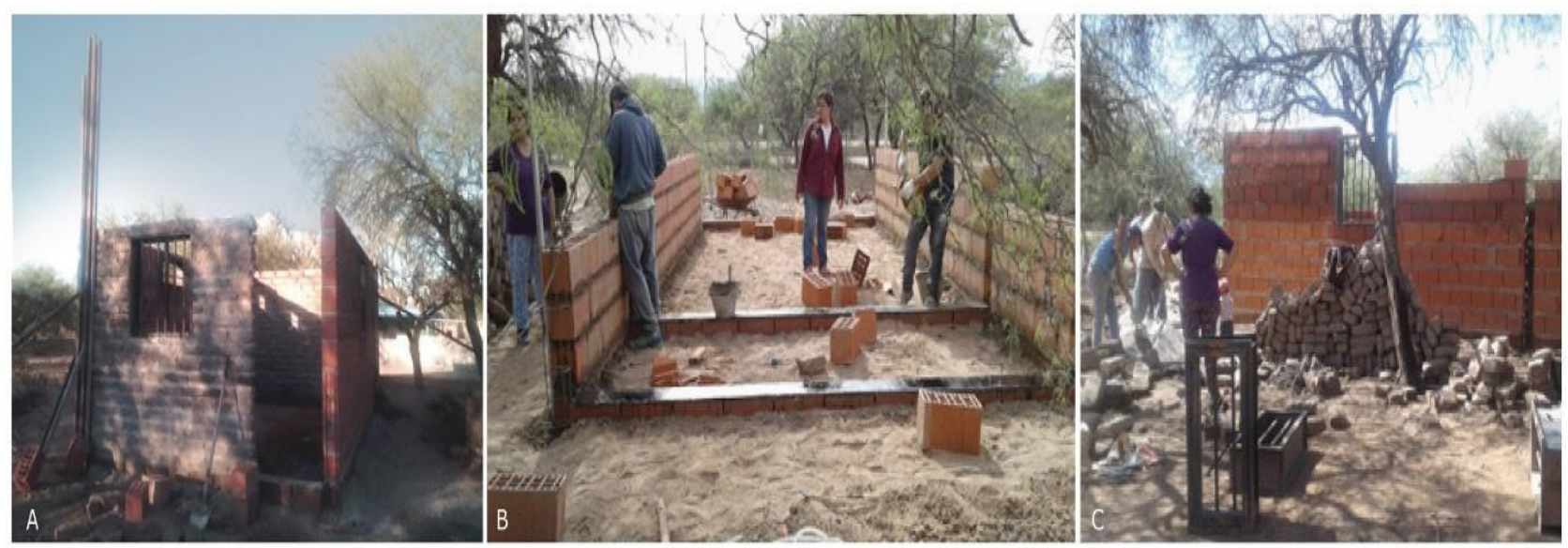

Figura 4 Resolución constructiva del frente (A), fundaciones y capa aisladora (B) y lateral con ladrillos y adobes (C)

resoluciones territoriales. En este mismo sentido se trabajó con las inscripciones ambientales propias del grupo campesino en diálogo con el equipo técnico condensando esos saberes ambientales para lograr un objetivo común: obtener una temperatura interior adecuada para la conservación de alimentos.

Asimismo, los referentes del Estado en la experiencia se inscribieron en una lógica de intervención diferente a las que usualmente despliegan, caracterizadas por un modo vincular lineal, jerárquico, con definiciones espaciales, tecnológicas y temporales unidireccionales.

Actualmente el proceso se encuentra en la etapa final de construcción. Esta práctica se acordó realizar por medio de la autoconstrucción, encausada a partir de un saber endógeno de la comunidad, considerando a la tarea de construir espacios como un trabajo "transmitido y conocido por todos, así como la producción de la manteca o de la harina para el consumo propio de la familia" (Lenzi, 2017, p.94). Consideramos que es aquí donde se logra condensar aún más la idea de autonomía en las decisiones tecnológicas del grupo. Los cimientos, estructura y cerramientos se realizaron en base a lo acordado en las etapas previas, sin embargo al momento de comenzar la construcción del techo aparecieron algunos interrogantes propios del grupo sobre cómo se comportaría este componente en relación a la amplitud térmica del lugar, al posible alojamiento de insectos, etc. A partir de esas dudas, el grupo definió colocar otro tipo de tecnología para el cielorraso con el fin de evitar posibles deterioros, expresando de esta manera el ejercicio autónomo en la toma de decisiones como grupo y también en las adecuaciones tecnológicas del sistema, lo cual habla de un manejo global del conocimiento al respecto del espacio diseñado (sin cajas negras, de saber experto).

\section{Conclusiones}

Como cierre del trabajo, daremos cuenta de algunas ideas fuerza que provocó la experiencia con el grupo Agua Viva de La Patria, INTA y MAASP, a fin de expresar cierto orden, desde lo más abstracto a lo más concreto.

En primer lugar, la perspectiva epistémica de la ecología de saberes, basada en conceptualizaciones de Boaventura de Sousa Santos y su llamado pensamiento postabismal, nos permitió imaginar una metodología de trabajo sensible a esos saberes territoriales en torno al hábitat campesino sistemáticamente silenciados, tanto desde las políticas públicas que alcanzan estos territorios como desde las prácticas académicas.

Tal como señalamos en el apartado de resultados, destacamos aquí una categoría central del trabajo de Santos: la co-presencia radical de saberes. Esta categoría se expresa en las posibilidades de desplegar otros modos de producción de conocimiento, basados en saberes emergentes de experiencias diversas (disciplinarios y también saberes tácitos), expresados a demás a través de soportes distintos: 
corporales, verbales, materiales, espaciales, vinculares. En la experiencia que se presentó, las posibilidades de cristalizar esto estuvieron dadas por el doble juego de desplazarnos (los actores miembros del sector científicotécnico) de los espacios pre-asignados (propios del esquema de extractivismo académico) y situarnos en experiencias en las que el soporte discursivo privilegiado pudiera ser uno distinto del verbocentrado. Las historias en el territorio, los silencios, las dudas, las voces autorizadas al interior del grupo, las jerarquizaciones según edad y/o género, etc. son una serie de reasignaciones que componen un proceso de producción de conocimiento sumamente singular, que no implica una romantización del ejercicio de producir conocimiento con otros/as pero sí una práctica eminentemente política, que procura reensamblar las formas vinculares establecidas desde la academia y sus estrategias de saber-poder. Esto último no implica desacreditar el conocimiento científico, simplemente implica pensarlo en contextos contrahegemónicos.

Como corolario de estos elementos, no es posible sostener procesos de producción de conocimiento orientado al desarrollo de tecnologías para el hábitat, campesino en este caso, en un ejercicio abstracto, sino como ejercicio situado, multivariable y transdisciplinar. Entonces, entendemos el desarrollo situado de tecnología, desde un enfoque integral, a partir del diálogo de saberes como estrategia de:

- praxis decoloniales, que permiten desarrollar tecnologías sociales inclusivas

- fortalecimiento identitario y de saberes comunitarios

- apoyo a formas de resistencia a las formas dominantes de la ruralidad-extractivista

- comprensión y apoyo al fortalecimiento de formas de habitar rural-campesino (residencia, económica-productiva y organizativa).

Un desafío importante de este equipo de investigación viene dado por la intención de aportar, desde estas experiencias, a la configuración de políticas públicas de hábitat campesino (entendido en su triple funcionalidad, antes referida) sensibles al territorio y a las prácticas de sus habitantes más vulnerados, aspectos que entendemos constituyen una perspectiva orientada al abordaje integral del hábitat.

\section{Referencias}

De Sousa, B(2009) Una epistemología del sur: la reivindicación del conocimiento y la emancipación social. México: Siglo XXI. CLACSO.

De Sousa, B. (2006) Conocer desde el Sur. Para una cultura política emancipatoria. Editorial Fondo Editorial de la Facultad de Ciencias Sociales. UNMSM Programa de Estudios sobre Democracia y Transformación Global. Solís. Madrid: Akal.

Garzón, L. (2011). “Técnicas mixtas”. En: Neves, C. y Faria, O. Técnicas de construcción con tierra. (pp. 62-71). Bauru-SP: FEB-UNESP/PROTERRA.

Grosfoguel, R. (2015). Del extractivismo económico al extractivismo epistémico y ontológico. Revista Internacional de Comunicación y Desarrollo, 4, 33-451

International Council on Monuments and Sites (ICOMOS) (1965). Carta internacional sobre la conservación y la restauración de monumentos y sitios. En: // Congreso Internacional de Arquitectos y Técnicos de Monumentos Históricos. Venecia, Italia: Consejo Internacional de Monumentos y Sitios (ICOMOS, su sigla en inglés).

Lenzi, C. (2017). La vivienda campesina en el programa Mi Casa Mi Vida (Brasil). Tesis de la Maestría 
en Arquitectura y Urbanismo de la Universidad de Sao Paulo.

Mignolo, W. (2003). Historias locales / diseños globales. Colonialidad, conocimientos subalternos y pensamiento fronterizo. Madrid: Akal.

Rolon, G., Olivarez, J., Dorado, P. \& Varela, G. (2016). "Las construcciones del espacio domiciliar y peridomiciliar rural como factores de riesgo de la enfermedad de Chagas". En: Construcción con Tierra. (pp. 57-68). Centro de Investigación Hábitat y Energía: Buenos Aires

Tomassi, J.; Bellmann, L. (2018). Bioarquitectura: diseño y construcción con tierra. Revista Estructuras FAUD, UNC, 1 (2).

Vasilachis, I. (2007). Estrategias de investigación cualitativa. Buenos Aires: Gedisa editoria.

\section{Fuentes estadísticas consultadas}

INDEC: http://www.indec.gob.ar/

DGEC: http://estadistica.cba.gov.ar/

\section{Agradecimientos}

Se agradece al Instituto Nacional de Tecnología Agropecuaria (INTA) a través del Proyecto Especial Prohuerta "El valor del monte", al Ministerio de Agua Ambiente y Servicios Públicos de la provincia de Córdoba (MAASP) y al Consejo Nacional de Investigaciones Científicas y Técnicas (CONICET). 